\title{
Simulation of Payne effect of elastomeric isolators with a harmonic balance method
}

\author{
V. Jaumouilléa, ${ }^{\mathrm{b}}$, J.-J. Sinou ${ }^{\mathrm{a}, *}$ and B. Petitjean ${ }^{\mathrm{b}}$ \\ ${ }^{a}$ Laboratoire de Tribologie et Dynamique des Systèmes UMR-CNRS 5513, Ecole Centrale de Lyon, Ecully Cedex, \\ France \\ ${ }^{\mathrm{b}}$ EADS Innovation Works, Suresnes, France
}

Received 27 June 2011

Revised 22 December 2011

\begin{abstract}
In the presented work, a non linear effect of rubber referred as Fletcher-Gent effect or Payne effect is investigated. It leads to a change in the rubber dynamic modulus with vibration amplitudes and, consequently, modifies resonance frequencies of mechanical systems including non linear elastomers. In this study a new methodology is developed to take into account Payne effect in a linear viscoelastic rubber material. Small vibration amplitudes around a no-preloaded state are predicted by considering frequency and amplitude dependencies of the material. This methodology has the advantage of using tabular experimental data from characterization tests which avoids the development of a complex model. In order to compute frequency responses, the non linear harmonic balance method is used and, for each iteration, new rubber properties are affected at each element according to its strain state. An equivalent strain measure is evaluated from the element strain energy density. This equivalent strain allows to associate dynamic properties of a material element subjected to multiaxial strain state with experimental dynamic properties of a material sample subjected to an uniaxial strain state. Practically, DMAP alter procedures are developed in order to evaluate energies in models defined with MSC.Nastran and the non linear solver is developed with Matlab. The method is applied on a satellite instrument isolator including four non linear rubber mounts. A non homogeneous spatial distribution of element equivalent strains is observed. Moreover, the maximum equivalent strain varies with frequency. These two observations validate the use of a specific methodology to deal with amplitude dependency of rubber.
\end{abstract}

Keywords: Harmonic balance method, viscoelasticity, Payne effect

\section{Introduction}

The use of rubber mounts is a common solution in industry in order to connect two structures and to achieve a combination of stiffness and vibration isolation. Kunz [1] applied a rubber isolator technology on helicopters and Bruger et al. [2] optimized comfort properties of elastomeric properties for automotive industry.

Simulations of rubber parts need to consider various complex effects and deal with properties varying with many parameters. Indeed, elastic and damping properties are strongly influenced by temperature and frequency (equivalence temperature-frequency), preload,... Many different experimental studies have been performed to study all these dependencies, more particularly on carbon-filled rubber which usually present a more complex behaviour [3, 4]. One frequently studied dependency is the amplitude dependency. Mullins effect [5] represents one of these effect where a softening effect is observed for the first excitation cycles on a rubber sample. A second effect, referred to Fletcher-Gent or Payne effect [6,7], is the dependency of the dynamic properties on strain amplitude when material

\footnotetext{
*Corresponding author: Jean-Jacques Sinou, Laboratoire de Tribologie et Dynamique des Systèmes UMR-CNRS 5513, Ecole Centrale de Lyon, 36 avenue Guy de Collongue, 69134 Ecully Cedex, France. E-mail: jean-jacques.sinou@ec-lyon.fr.
} 
is submitted to small dynamic vibrations. Origin of Payne effect is not clearly established but supposed to be a continuous breaking and reforming Van der Waals forces between carbon-black aggregates [8].

Concerning the modelling of rubber, frequency dependent behaviour is today quite well modeled through the use of simple models involving combinations of springs and dashpots [9,10]. For a full three dimensional case, hereditary integrals are used to formulate constitutive equations [11]. Furthermore, fractional derivative models have also been developed to obtain a better frequency-dependent behaviour and to reduce the number of parameters to identify [12]. Nevertheless, as regards the amplitude dependent behaviour modeling, more complex models have to be established for filled rubbers. The first one-dimensional model is the Kraus model [13] who dealt with non linear viscous forces. Kraus considered contacts and shocks between aggregates. Later, Ulmer [14] improved this model to obtain a better approach of experimental observations. Other models tried to represent the irreversible slip processes occurring between filler particles by introducing friction elements [15-17]. These models are often limited to a one-dimensional case or are difficult to use in a frequency domain. Austrell et al. [18] made use of elastoplasticity elements to simplify implementation. The main drawback of these approaches is that a parameter identification is needed in conjunction with specific tests and that these models are limited to the time domain. For frequency analysis, these models are not very well adapted. However, some methodologies have been introduced by Gil-Negrete et al. [19] who proposed to apply a first quasi-static step on a studied mount submitted to a given amplitude to assign adapted dynamic properties on each element according to its strain state in order to predict in a second time the dynamic stiffness of the mount at this same dynamic amplitude. Then, Rabkin [20] proposed to predict the dynamic stiffness of a rubber sample submitted to small vibrations around a preloaded state by modifying UPHI subroutine in a Marc analysis.

This paper proposes to investigate this non linear Payne effect of rubber and develop a methodology to predict frequency responses of a complete system including non linear rubber mounts. This method does not include any parametric model and use tabular experimental data from a characterization test on a rubber sample. This approach uses a non linear harmonic balance method to deal with amplitude dependency. Only first harmonic is retained here due to practical observation [21]. However, more harmonics may be used in case of stronger non linearities. The technique is based on the calculation on an equivalent strain for each element and for each iteration in order to assign appropriate rubber dynamic properties. Indeed, each element of the rubber parts does not have the same strain state and consequently the same rubber property. This methodology is applied for models defined with MSC.Nastran and resolution is performed by the use of DMAP alter procedures and Matlab routines. Finally, it can be noted that the use of harmonic balance for problems in which the terms depend on the frequency and amplitude has been studied for the particular case of viscoelastic materials by Bilasse et al. [22]: the authors studied the nonlinear vibration of viscoelastic shell structures by coupling an approximated harmonic balance method with one mode Galerkin's procedure. In [23], they proposed numerical models with linear and nonlinear vibrations analyses of viscoelastic sandwich beams with various viscoelastic frequency dependent laws.

The paper is organized as follows: method description is detailed in a first section. One application case is presented in a second section. In this application, a satellite instrument isolator including four rubber mounts is harmonically excited. Results are then shown to highlight the non linear behaviour of the system and the ability of the proposed technique to represent a non homogeneous spatial distribution of element strains.

\section{Methodology}

This methodology proposes a new procedure to consider Payne effect for material modeling of viscoelastic materials in FE models with MSC.Nastran for small amplitude harmonic vibrations. Materials considered in this study are supposed to be isotropic, isothermal and nearly incompressible.

\subsection{Viscoelasticity in MSC.Nastran for frequency analysis}

For small amplitude strains, MSC.Nastran provides the ability to represent the behaviour of a viscoelastic material in the frame of the linear viscoelastic theory with a complex frequency-dependent shear modulus:

$$
G(f)=G^{\prime}(f)+i G^{\prime \prime}(f)
$$


where $G^{\prime}$ and $G^{\prime \prime}$ represent respectively the shear storage and loss moduli.

A viscoelastic behaviour leads to a complex dynamic stiffness matrix for all viscoelastic elements of the model. Stiffness matrix is computed on the basis of a representative reference modulus $G_{r e f}$ which often corresponds to the long-term quasi-static shear modulus $G_{0}=\lim _{\omega \rightarrow 0} G(\omega)$. $G_{r e f}$ is independent of frequency. Thus complex stiffness matrix for viscoelastic elements is expressed as:

$$
\mathbf{K}=\left(\frac{G^{\prime}(f)+i G^{\prime \prime}(f)}{G_{r e f}}\right) \mathbf{K}_{r e f}
$$

where $\mathbf{K}_{\text {ref }}$ is the stiffness matrix with $G_{r e f}$ as shear modulus.

Additionally, a structural damping coefficient $g$ may be affected on the whole model. A structural damping coefficient $g_{\text {ref }}$ must also be defined on the viscoelastic element group. To combine these two conditions, MSC.Nastran uses two tabular functions $T R(f)$ and $T I(f)$ to represent the complex moduli of all viscoelastic materials. The calculation of the complex stiffness matrix is then given by:

$$
\mathbf{K}=\left(\left(1+g_{\text {ref }} T R(f)\right)+i\left(g+g_{r e f} T I(f)\right)\right) \mathbf{K}_{r e f}
$$

To assure equivalence with Eq. (2), $T R(f)$ and $T I(f)$ are expressed as:

$$
\begin{aligned}
& T R(f)=\frac{1}{g_{\text {ref }}}\left[\frac{G^{\prime}(f)}{G_{r e f}}-1\right] \\
& T I(f)=\frac{1}{g_{\text {ref }}}\left[\frac{G^{\prime \prime}(f)}{G_{\text {ref }}}-g\right]
\end{aligned}
$$

MSC.Nastran does not provide the ability to define different dynamic properties for each particular viscoelastic element. When a viscoelastic material with amplitude dependent properties is subjected to a non homogeneous strain field, it may lead to severe errors for the dynamic stiffness matrix calculation.

\subsection{Adaptation to Payne effect}

The amplitude dependence of the complex shear modulus for small vibrations is referred to Payne effect and is noticeable for filled elastomers. This complex non linear effect is often attributed to irreversible slip processes between filler particles. Consequently, models of linear viscoelasticity are not suitable for frequency analysis. Based on an analogy with microplastic theory, some rheological models have been developed which introduce friction elements $[15,16]$. These models are often formulated for uniaxial cases and are not adapted for multiaxial rubber models. Moreover a complex parameter identification has to be lead on material samples in terms of test conditions and parameter calculations. Up to now FE commercial codes do not provide the possibility to easily model Payne effect for frequency analysis.

An approximate procedure has been developed for dynamic analysis. This procedure has the advantage of using tabular experimental data from simple characterization tests (shear or compression tests) on material specimens and does not need any parameter identification for some simplified models. The non linear Harmonic Balance Method (HBM) is used to calculate frequency responses for a FE model integrating non linear viscoelastic elements (in terms of frequency and amplitude dependent shear modulus) subjected to an external dynamic load. Vibration amplitudes are not a priori known so equations have to be solved iteratively.

For each iteration of the solver, strain energy density is first computed element-wise and for the previous displacement field. Then, a local equivalent strain $\gamma_{e q}^{e}$ is deduced so that the element strain energy density is equal to the homogeneous strain energy density in a characterization sample tested at the same dynamic amplitude $\gamma_{e q}^{e}$. This energy balance enables to associate dynamic properties of a material element subjected to multiaxial strain state with experimental dynamic properties of a material sample subjected to an uniaxial strain state. Afterwards, shear storage and loss moduli associated with this equivalent strain are deduced from experimental data and assembly of all elementary complex stiffness matrices is performed. A new displacement field is finally calculated from HBM equations and a non linear solver repeats this operation until convergence.

In the following, HBM formulation is first presented in order to secondly detail the evaluation of non linear forces generated by all viscoelastic elements of the FE model. 


\subsubsection{HBM formulation}

In order to compute frequency responses of a mechanical system integrating viscoelastic elements with amplitude dependent properties, HBM method is used. This method is able to compute periodic responses of non linear systems submitted to an external loading [24] or to self-excited vibrations [25]. HBM is also able to compute quasi-periodic responses [26] and able to conduct stability analysis [27]. In the following, basic equations are recalled and a non linear solver is used for convergence. More details are given in Appendix 1 for the interested reader. Condensation techniques and continuation may also be applied in order to accelerate calculation.

First we consider a discrete mechanical system with $n$ degrees of freedoms (dofs) described with its $n \times n$ mass matrix $\mathbf{M}$, stiffness matrix $\mathbf{K}$ and damping matrix $\mathbf{D}$. The system includes non linear viscoelastic elements and $v$ dofs are linked to these elements. Mass of all viscoelastic elements are integrated in the global mass matrix M. D and $\mathbf{K}$ include all linear parts of the model. Forces generated by the complex dynamic stiffness of all viscoelastic elements $\mathbf{K}_{v}^{\mathbb{C}}=\mathbf{K}_{v}^{\prime}+i \mathbf{K}_{v}^{\prime \prime}$ are considered as an external non linear force $F_{N L}(X, \dot{X}, \Omega, t)$ which depends on degrees of freedom displacements $X$, velocities $\dot{X}$, time $t$ and angular frequency $\Omega$. $\Omega$ is the angular frequency of an external periodic force $F_{L}(\Omega, t)$ applied to the system. The governing equation of motion may be written as:

$$
\mathbf{M} \ddot{X}+\mathbf{D} \dot{X}+\mathbf{K} X=F(X, \dot{X}, \Omega, t)=F_{L}(\Omega, t)+F_{N L}(X, \dot{X}, \Omega, t)
$$

Then, we assume that the non-linear dynamical response of the system can be approximated by finite Fourier series:

$$
\begin{aligned}
X(t)= & B_{0}+\sum_{k=1}^{m}\left(A_{k} \sin \left(\frac{k}{\nu} \Omega t\right)+B_{k} \cos \left(\frac{k}{\nu} \Omega t\right)\right) \\
X(t)= & \mathbf{T}(t) Z=\left[\mathbf{I} \sin \left(\frac{\Omega}{\nu} t\right) \mathbf{I} \cos \left(\frac{\Omega}{\nu} t\right) \mathbf{I} \ldots \sin \left(\frac{k}{\nu} \Omega t\right) \mathbf{I} \cos \left(\frac{k}{\nu} \Omega t\right) \mathbf{I} \ldots\right] \\
& {\left[B_{0} A_{1} B_{1} \ldots A_{k} B_{k} \ldots\right]^{T} }
\end{aligned}
$$

where $\mathbf{I}$ is the $n \times n$ identity matrix, $Z=\left[B_{0} A_{1} B_{1} \ldots A_{k} B_{k} \ldots\right]^{T}$ is the $(2 m+1) n \times 1$ vector containing Fourier coefficients, $m$ is the number of harmonics retained for the truncation, $\nu$ is an integer used to represent possible subharmonics, and $\mathbf{T}(t)=\left[\mathbf{I} \sin \left(\frac{\Omega}{\nu} t\right) \mathbf{I} \cos \left(\frac{\Omega}{\nu} t\right) \mathbf{I} \ldots \sin \left(\frac{k}{\nu} \Omega t\right) \mathbf{I} \cos \left(\frac{k}{\nu} \Omega t\right) \mathbf{I} \ldots\right]$ is the $n \times(2 m+1) n$ matrix containing trigonometric functions. Finally, we assume that the vector force $F_{N L}(X, \dot{X}, \Omega, t)$ can be solved in finite Fourier series (see Appendix 1 for more details). Generally, the number of harmonic coefficients is selected on the basis of the number of significant harmonics expected in the non-linear dynamical response. Concerning Payne effect in viscoelastic materials, it has been described that, within certain strain amplitude limit, higher-order terms are not significant in the material response. The reader is referred to Roland [21] for more details. Consequently, a single harmonic has been retained in the HBM procedure leading to the following equation system (see Appendix 1 for more details):

$$
\left[\begin{array}{cc}
\mathbf{K}-\Omega^{2} \mathbf{M} & -\Omega \mathbf{D} \\
\Omega \mathbf{D} & \mathbf{K}-\Omega^{2} \mathbf{M}
\end{array}\right]\left[\begin{array}{l}
A_{1} \\
B_{1}
\end{array}\right]=b_{L}(\Omega)+b_{N L}\left(Z_{v}, \Omega\right)
$$

where $\mathbf{M}, \mathbf{K}$ and $\mathbf{D}$ are the mass, stiffness and damping matrices. $b_{L}(\Omega)$ defines the Fourier coefficients from an external periodic force applied to the system. $\Omega$ is the angular frequency of the external periodic force. $b_{N L}\left(Z_{v}, \Omega\right)$ defines the Fourier coefficients from the non linear force $F_{N L}(X, \dot{X}, \Omega, t)$. It depends only on the Fourier coefficients $Z_{v}$ of the $v$ degrees of freedom linked with the viscoelastic elements.

\subsubsection{Non linear force evaluation}

In order to calculate Fourier coefficients of non linear forces $b_{N L}$, the non linear force $F_{N L}$ has to be detailed. Indeed, dofs are reorganized into $v$ dofs which are linked to viscoelastic elements and $n v$ dofs which are not using a boolean transition matrix $\mathbf{P}$ :

$$
X=\mathbf{P}\left[\begin{array}{c}
X_{n v} \\
X_{v}
\end{array}\right]
$$


Thus, the complex dynamic stiffness matrix of all viscoelastic elements which is function of $X_{v}$, may be expressed as:

$$
\mathbf{K}_{v}^{\mathbb{C}}\left(X_{v}, \Omega\right)=\mathbf{K}_{v}^{\prime}\left(X_{v}, \Omega\right)+i \mathbf{K}_{v}^{\prime \prime}\left(X_{v}, \Omega\right)
$$

Thus, one may write:

$$
F_{N L}(X, \dot{X}, \Omega, t)=-\left[\begin{array}{cc}
\mathbf{0} & \mathbf{0} \\
\mathbf{0} & \mathbf{K}_{v}^{\prime}\left(X_{v}, \Omega\right)
\end{array}\right]\left[\begin{array}{c}
X_{n v} \\
X_{v}
\end{array}\right]-\left[\begin{array}{cc}
\mathbf{0} & \mathbf{0} \\
\mathbf{0} & \frac{\mathbf{K}_{v}^{\prime \prime}}{\Omega}\left(X_{v}, \Omega\right)
\end{array}\right]\left[\begin{array}{c}
\dot{X}_{n v} \\
\dot{X}_{v}
\end{array}\right]
$$

This stiffness matrix depends on the strain amplitude in the material and does not vary over one period. This remark allows to calculate non linear Fourier coefficients with the relation:

$$
\begin{aligned}
& b_{N L}=\frac{1}{T} \int_{0}^{T} T \mathbf{T}(t) F_{N L}(X, \dot{X}, \Omega, t) d t \\
& b_{N L}=-\left[\begin{array}{cccc}
\mathbf{0} & \mathbf{0} & \mathbf{0} & \mathbf{0} \\
\mathbf{0} & \mathbf{K}_{v}^{\prime}\left(Z_{v}, \Omega\right) & \mathbf{0} & \mathbf{0} \\
\mathbf{0} & \mathbf{0} & \mathbf{0} & \mathbf{0} \\
\mathbf{0} & \mathbf{0} & \mathbf{0} & \mathbf{K}_{v}^{\prime}\left(Z_{v}, \Omega\right)
\end{array}\right] Z-\left[\begin{array}{cccc}
\mathbf{0} & \mathbf{0} & \mathbf{0} & \mathbf{0} \\
\mathbf{0} & \frac{\mathbf{K}_{v}^{\prime \prime}}{\Omega}\left(Z_{v}, \Omega\right) & \mathbf{0} & \mathbf{0} \\
\mathbf{0} & \mathbf{0} & \mathbf{0} & \mathbf{0} \\
\mathbf{0} & \mathbf{0} & \mathbf{0} & \frac{\mathbf{K}_{v}^{\prime \prime}}{\Omega}\left(Z_{v}, \Omega\right)
\end{array}\right] \nabla Z \\
& {\left[\begin{array}{c}
S_{N L n v} \\
S_{N L v} \\
C_{N L n v} \\
C_{N L v}
\end{array}\right]=-\left[\begin{array}{cccc}
\mathbf{0} & \mathbf{0} & \mathbf{0} & \mathbf{0} \\
\mathbf{0} & \mathbf{K}_{v}^{\prime}\left(Z_{v}, \Omega\right) & \mathbf{0} & -\frac{\mathbf{K}_{v}^{\prime \prime}}{\Omega}\left(Z_{v}, \Omega\right) \\
\mathbf{0} & \mathbf{0} & \mathbf{0} & \mathbf{0} \\
\mathbf{0} & \frac{\mathbf{K}_{v}^{\prime \prime}\left(Z_{v}, \Omega\right)}{\Omega} & \mathbf{0} & \mathbf{K}_{v}^{\prime}\left(Z_{v}, \Omega\right)
\end{array}\right]\left[\begin{array}{c}
A_{1 n v} \\
A_{1 v} \\
B_{1 n v} \\
B_{1 v}
\end{array}\right] }
\end{aligned}
$$

Note that $Z_{v}=\left[A_{1 v} B_{1 v}\right]^{T}$ represents the Fourier coefficients of the $v$ dofs in relation with all viscoelastic elements.

Due to the amplitude-dependence of the stiffness matrix of all viscoelastic elements, a non linear solver is used. From a displacement field ${ }^{(i)} Z$ obtained after iteration $i$, a new value of the viscoelastic dynamic stiffness matrix ${ }^{(i)} \mathbf{K}_{v}\left({ }^{(i)} Z_{v}, f\right)$ is performed in order to deduce a new value for the non linear forces ${ }^{(i)} b_{N L}$.

The strain field is not necessarily homogeneous in all the viscoelastic part leading to different material properties depending on the considered point. The new developed procedure is able to take into consideration this effect by affecting different material properties for each viscoelastic element. For an element $e$ of the set $V$ of all viscoelastic elements, an elementary matrix ${ }^{(i)} \mathbf{K}_{v}^{e}\left({ }^{(i)} Z_{v}^{e}, f\right)$ is calculated from the displacement field ${ }^{(i)} Z_{v}^{e}$ associated to this element. All these elementary matrices are then assembled to deduce the complete dynamic stiffness matrix ${ }^{(i)} \mathbf{K}_{v}\left({ }^{(i)} Z_{v}, f\right)$ :

$$
{ }^{(i)} \mathbf{K}_{v}\left({ }^{(i)} Z_{v}, f\right)=\sum_{e \in V}{ }^{(i)} \mathbf{K}_{v}^{e}\left({ }^{(i)} Z_{v}^{e}, f\right)
$$

In the following, we detail the calculation of an elementary matrix ${ }^{(i)} \mathbf{K}_{v}^{e}\left({ }^{(i)} Z_{v}^{e}, f\right)$ from an initial displacement field ${ }^{(i)} Z_{v}^{e}$.

\subsubsection{Strain energy density}

In the following, index $i$ will be remove for simplification. All relations remain valid for each iteration.

For each viscoelastic element, an equivalent strain measure $\gamma_{e q}^{e}$ is computed from an elementary displacement field $Z_{v}^{e}$. It allows to associate dynamic properties $G^{\prime e}$ and $G^{\prime \prime e}$ coming from an uniaxial test with the strain state in the element which combines different sollicitations.

The strain equivalent strain is deduced at each iteration from the elementary displacement field $Z_{v}^{e}$ and from an evaluation of the strain energy in an element calculated with a real shear modulus equal to $G_{\text {ref }}$. This strain energy may be expressed as: 


$$
\begin{aligned}
& E^{e}(t)=\frac{1}{2}{ }^{T} X_{v}^{e}(t) \mathbf{K}_{r e f} X_{v}^{e}(t) \\
& E^{e}(t)=\frac{1}{2} Z_{v}^{e T} \mathbf{T}(t) \mathbf{T}(t) \mathbf{N}_{\mathbf{K}_{r e f}} Z_{v}^{e} \\
& E^{e}(t)=\frac{1}{2} Z_{v}^{e}\left[\begin{array}{cc}
\sin ^{2}(\Omega t) \mathbf{I} & \sin (\Omega t) \cos (\Omega t) \mathbf{I} \\
\sin (\Omega t) \cos (\Omega t) \mathbf{I} & \cos ^{2}(\Omega t) \mathbf{I}
\end{array}\right] \mathbf{N}_{\mathbf{K}_{r e f} Z_{v}^{e}} \\
& E^{e}(t)=\frac{1}{2} Z_{v}^{e}\left[\begin{array}{cc}
\frac{1-\sin (2 \Omega t)}{2} \mathbf{I} & \frac{\sin (2 \Omega t)}{2} \mathbf{I} \\
\frac{\sin (\Omega t)}{2} \mathbf{I} & \frac{1+\cos (2 \Omega t)}{2} \mathbf{I}
\end{array}\right] \mathbf{N}_{\mathbf{K}_{r e f} Z_{v}^{e}} \\
& E^{e}(t)=\frac{1}{4}{ }^{T} Z_{v}^{e} \mathbf{N}_{\mathbf{K}_{r e f} Z_{v}^{e}+\frac{1}{4}\left[\left({ }^{T} B_{1 v}^{e} \mathbf{K}_{r e f} B_{1 v}^{e}-{ }^{T} A_{1 v}^{e} \mathbf{K}_{r e f} A_{1 v}^{e}\right) \cos (2 \Omega t)+\left(2^{T} A_{1 v}^{e} \mathbf{K}_{r e f} B_{1 v}^{e}\right) \sin (2 \Omega t)\right]}
\end{aligned}
$$

Thus strain energy in the element is composed of a constant term $E_{0}^{e}=\frac{1}{4}^{T} Z_{v}^{e} \mathbf{N}_{\mathbf{K}} Z_{r e f}^{e}$ which represents the mean value over one period and of an oscillatory term at the frequency $2 \Omega$. The amplitude of the term is:

$$
E_{a}^{e}=\frac{1}{4} \sqrt{\left({ }^{T} B_{1 v}^{e} \mathbf{K}_{r e f} B_{1 v}^{e}-{ }^{T} A_{1 v}^{e} \mathbf{K}_{r e f} A_{1 v}^{e}\right)^{2}+\left(2^{T} A_{1 v}^{e} \mathbf{K}_{r e f} B_{1 v}^{e}\right)^{2}}
$$

The peak value for each element over one period is consequently $E_{p e a k}^{e}=E_{0}^{e}+E_{a}^{e}$. The last step necessary for the evaluation of the equivalent strain is the computation of the strain energy density $\rho^{e} . \rho^{e}$ is classically obtained by dividing $E_{\text {peak }}^{e}$ by the element volume.

Practically, MSC.Nastran is able to provide strain energy and strain energy density for each element of the model for a frequency analysis so strain energy density is obtained for all viscoelastic after introduction of a complex displacement field corresponding with $Z_{v}$ values at each iteration. An alter procedure in DMAP language is written to perform these operations.

\subsubsection{Equivalent strain measure}

Experimental data usually come from a simple shear or compression test and results in a table providing complex dynamic modulus depending on frequency and on a single dynamic strain amplitude $\gamma_{\exp }$. The strain energy density calculated at the previous step will allow to estimate an equivalent strain measure for each element $\gamma_{e q}^{e}$. This equivalent strain is comparable with the experimental strain amplitude and will allow to deduce appropriate storage and loss moduli to apply in each element.

A energy balance is performed for each element by equalizing the obtained strain energy density with the homogeneous strain energy density in a characterization sample tested at a dynamic amplitude $\gamma_{e q}^{e}$. This amplitude is the equivalent strain amplitude which will be comparable with the experimental one $\gamma_{\text {exp }}$. It has been shown in Gil-Negrete et al. [19] that, for a material element subjected to its principal stresses, the deviatoric part of the total strain energy density may be expressed with the principal strains namely:

$$
\rho_{d}=\frac{G}{3}\left[\left(\epsilon_{p 1}-\epsilon_{p 2}\right)^{2}+\left(\epsilon_{p 2}-\epsilon_{p 3}\right)^{2}+\left(\epsilon_{p 3}-\epsilon_{p 1}\right)^{2}\right]
$$

For our study, experimental data are associated with a compression test. Consequently, for a cylinder material element compressed with a strain amplitude of $\gamma$ and with a shear modulus $G_{r e f}$, principal strains may be expressed as $\epsilon_{p 1}=\gamma$ and $\epsilon_{p 2,3}=-\nu \epsilon_{p 1}=-\frac{\gamma}{2}$ for a quasi incompressible material. In this case, distortion energy becomes:

$$
\begin{aligned}
\rho_{d T C} & =\frac{G_{r e f}}{3}\left[\left(\frac{3}{2} \gamma\right)^{2}+0+\left(\frac{3}{2} \gamma\right)^{2}\right] \\
\rho_{d T C} & =\frac{3 G_{r e f}}{2} \gamma^{2}
\end{aligned}
$$

Energy density balance is then written for each element to deduce an equivalent strain measure: 


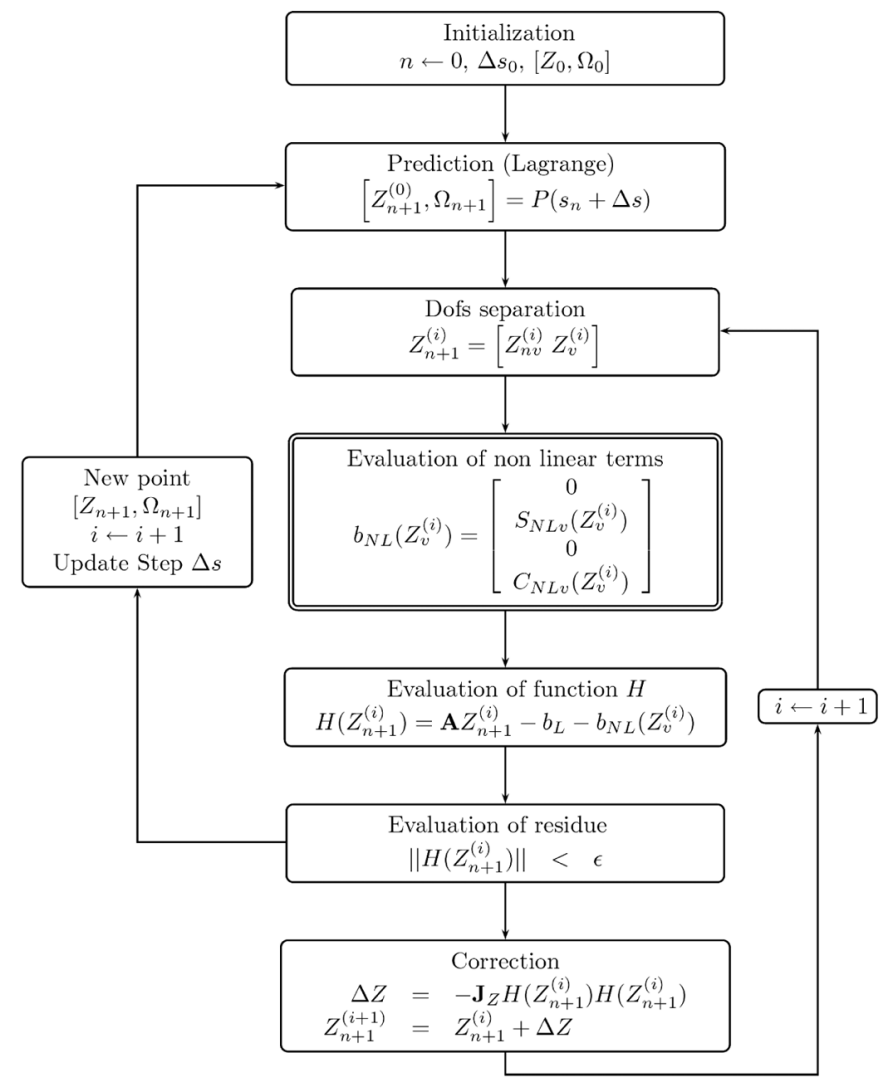

Fig. 1. Complete HBM algorithm with non term procedure.

$$
\begin{aligned}
\rho_{d T C}^{e} & =\rho^{e} \\
\frac{3 G_{r e f}}{2} \gamma_{e q}^{e 2} & =\rho^{e} \\
\gamma_{e q}^{e} & =\sqrt{\frac{2 \rho^{e}}{3 G_{r e f}}}
\end{aligned}
$$

This procedure is an approximate procedure since the reference modulus is used to evaluate the equivalent strain. This choice has been made to keep a realistic value. Moreover, it has been shown with a virtual test on a material sample under compression that equivalent strain measure evaluated with the above procedure is equal to the homogeneous strain obtained in the material for this compression test. Then, a purely kinematic relation is not used as in Gil-Negrete et al. [19] by convenience because MSC.Nastran provides the possibility to directly extract strain energy density of each element, avoiding to evaluate principal strains and averaging strain energy densities on all Gauss points. Finally, a dynamic calculation is performed to evaluate the equivalent strain instead of a quasi-static test in [19] which leads to a better representativity on a larger frequency band.

\subsubsection{Dynamic stiffness matrix assembly}

From experimental tabular data, storage $G^{\prime e}\left(\gamma_{e q}^{e}, f\right)$ and loss $G^{\prime \prime e}\left(\gamma_{e q}^{e}, f\right)$ moduli are deduced using the equivalent strain measure and the frequency. Quadratic and cubic interpolations are employed to deduce approximate values for an equivalent strain and a frequency which do not exactly match experimental datas. This step is performed with Matlab.

By analogy with Eq. (2), each elementary matrix is calculated with the relation: 


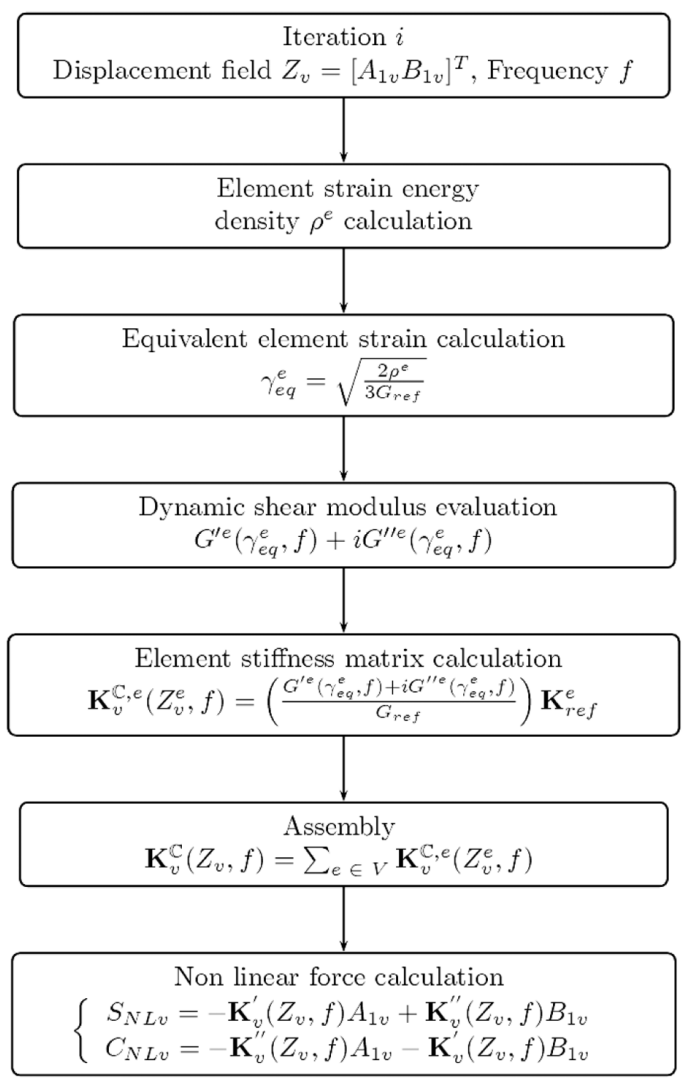

Fig. 2. Non linear force calculation for each iteration $i$.

$$
\begin{aligned}
& \mathbf{K}_{v}^{e}\left(Z_{v}^{e}, f\right)=\mathbf{K}_{v}^{e}\left(\gamma_{e q}^{e}, f\right) \\
& \mathbf{K}_{v}^{e}\left(Z_{v}^{e}, f\right)=\left(\frac{G^{\prime e}\left(\gamma_{e q}^{e}, f\right)+i G^{\prime \prime e}\left(\gamma_{e q}^{e}, f\right)}{G_{r e f}}\right) \mathbf{K}_{r e f}^{e}
\end{aligned}
$$

The final complex stiffness matrix of all viscoelastic elements $\mathbf{K}_{v}\left(Z_{v}, f\right)$ is finally assembled and Fourier coefficients of the non linear force $b_{N L}$ is then computed according to Eq. (12).

For practical convenience, calculation of stiffness complex matrix and Fourier coefficients is performed with an alter procedure with MSC.Nastran which easily deals with connectivity of the model.

\subsubsection{Practical procedure}

The complete algorithm with the different continuation steps is presented in Fig. 1. The algorithm is described in the particular case of a parameterized continuation with an curvilinear abscissa, a prediction with Lagrange polynomials and a Newton-Raphson correction method.

This procedure deals with the specific Payne effect in the "non linear term calculation". These detailed steps are then described in Fig. 2. As seen previously, resolution has to be made incrementally. From an displacement field (i) $Z_{v}$ obtained at the iteration $i$, main steps to deduce the non linear force Fourier coefficients are synthesized here:

1. ${ }^{(i)} Z_{v}$ : displacement field of all viscoelastic elements obtained for iteration $i$.

2. Calculation of the strain energy density ${ }^{(i)} \rho^{e}$ (peak value) for each element. Shear modulus is equal to the reference modulus $G_{r e f}$

3. Calculation of an equivalent strain measure ${ }^{(i)} \gamma_{e q}^{e}=\sqrt{\frac{2^{(i)} \rho^{e}}{3 G_{r e f}}}$ with an energy balance. 


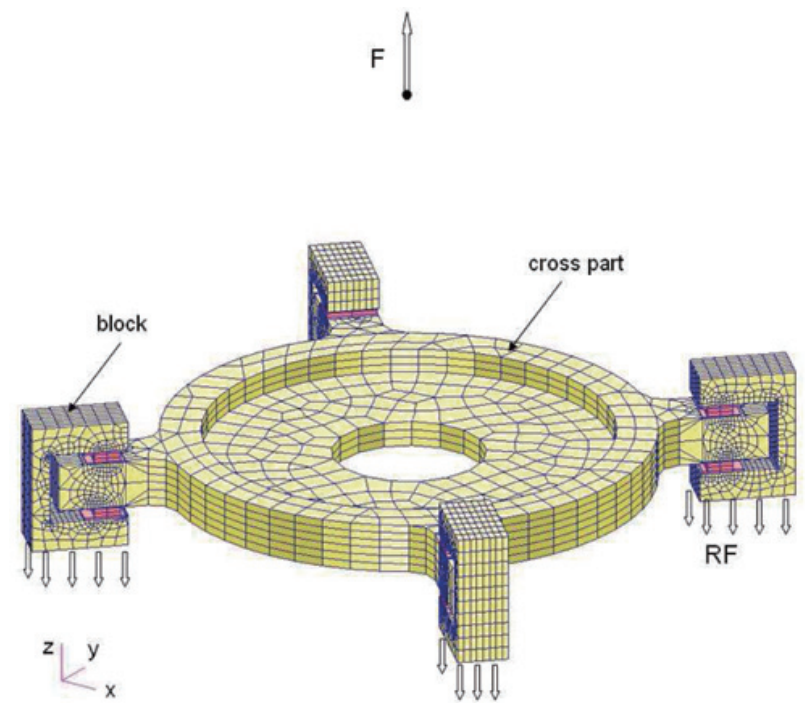

(a)

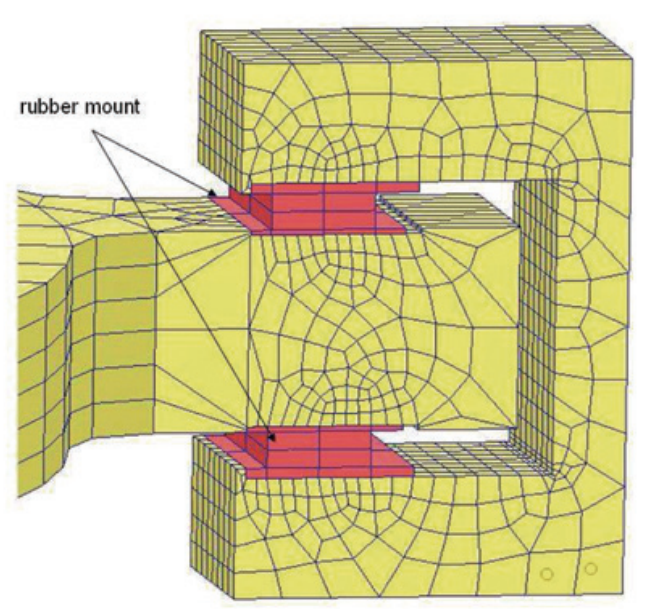

(b)

Fig. 3. Elastomeric isolator: (a) Global view, (b) Zoom on one rubber part. Dark colour: rubber

4. Evaluation of storage ${ }^{(i)} G^{\prime e}\left({ }^{(i)} \gamma_{e q}^{e}, f\right)$ and loss moduli ${ }^{(i)} G^{\prime \prime e}\left({ }^{(i)} \gamma_{e q}^{e}, f\right)$ with experimental data.

5. Calculation of each element complex stiffness matrix ${ }^{(i)} \mathbf{K}_{v}^{e}\left({ }^{(i)} Z_{v}^{e}, f\right)$.

6. Assembly of the complex stiffness matrix of all viscoelastic element ${ }^{(i)} \mathbf{K}_{v}\left({ }^{(i)} Z_{v}, f\right)$.

7. Calculation of non linear force Fourier coefficients ${ }^{(i)} A_{1 v}$ and ${ }^{(i)} B_{1 v}$.

It has also to be noted that this procedure is used for jacobian matrix calculation. Steps $\mathrm{n}^{\circ} 1,3,4$ are performed with Matlab whereas steps $n^{\circ}$ 2,5,6,7 are performed with an alter DMAP procedure using with MSC.Nastran.

\section{Application case}

\subsection{Elastomeric isolator}

An application is carried out on an elastomeric isolator from space industry. This system is designed to isolate a satellite instrument. Four rubber mounts are inserted between four metallic (aluminium) blocks clamped on the satellite and a circular part referred as "cross part" on which specific instrument is mounted. Figure 3 shows a detailed view of the considered structure. Satellite instrument is only represented here by a concentrated mass and does not appear on the figure. Weight of mounted instrument is approximatively $16 \mathrm{~kg}$. The global weight of the structure including isolator and instrument is nearly $20 \mathrm{~kg}$. Height of the structure is $180 \mathrm{~mm}$ and cross diameter is $200 \mathrm{~mm}$. The four rubber mounts integrates two plane-parallel areas whom dimensions are $20 \times 10 \times 3.5 \mathrm{~mm}$.

Elastomeric parts are made of a rubber which presents a pronounced amplitude dependency. Material characterization has been performed for dynamic strain amplitudes of $\pm 0.1 \%, \pm 0.25 \%, \pm 1 \%, \pm 2 \%$ and $\pm 5 \%$. Tested frequency bands are $1-185 \mathrm{~Hz}$ for $\pm 5 \%, 1-395 \mathrm{~Hz}$ for $\pm 2 \%, 1-500 \mathrm{~Hz}$ for $\pm 0.1 \%, \pm 0.25 \%$ and $\pm 1 \%$. For simulations, only experimental data for a frequency band of $1-185 \mathrm{~Hz}$ will be considered in order to have material data for all dynamic amplitudes. In order to illustrate frequency and amplitude dependencies, experimental storage modulus is shown in Fig. 4. Values have been removed for confidentiality.

Isolator performance will be quantified with a force transmissibility. Indeed, for a given excitation force amplitude applied on the satellite instrument, reaction forces at the base of the four metallic blocks are observed. These blocks are clamped on the primary structure. For simulation, they are fixed. Force transmissibility is then deduced for different excitation configurations and maximum is observed for resonances. The main difficulty is the ability to 


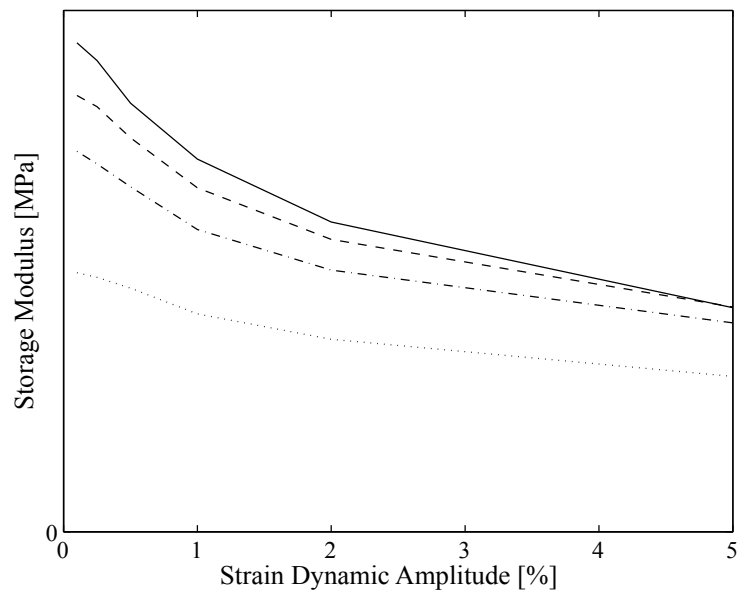

(a)

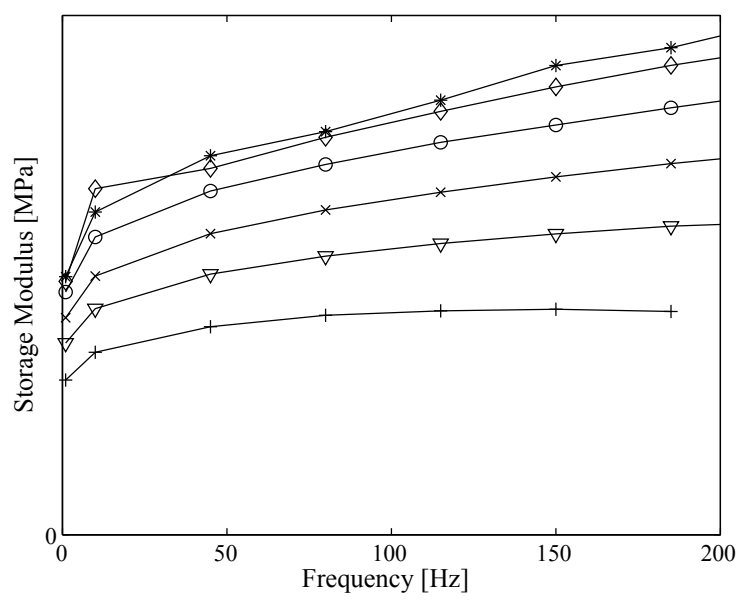

(b)

Fig. 4. Storage modulus: (a) Amplitude dependency; - (1 Hz); - - $(45 \mathrm{~Hz}) ;-$-. $(115 \mathrm{~Hz}) ; \ldots$ (.. $185 \mathrm{~Hz})(\mathrm{b})$ Frequency dependency; * $(0.1 \% \mathrm{~Hz}) ; \diamond$ $(0.25 \%) ; \bigcirc(0.5 \%) ; \times(1 \%) ; \nabla(2 \%) ;+(5 \%)$.

predict resonance frequency and vibration amplitude due to the non linear stiffness of rubber mounts. This study focuses on the pumping mode so excitation is applied vertically on the satellite instrument. Consequently, rubber mounts are submitted to tension/compression.

\subsection{Numerical model}

A numerical model of the system is built with MSC.Nastran and is shown in Fig. 3. This model is not initially submitted to a static preload. The satellite instrument is modelled as a single concentrated mass and an inertia matrix located at its center of gravity. This mass is rigidly connected to the upper surface of the cross part through rigid body elements which are not represented on Fig. 3. Cross part and blocks are discretized with 10739 four-sided CTETRA and six-sided CHEXA solid elements.

The geometry of elastomeric parts has been simplified in order to neglect elastomeric areas which are not significantly deformed. It has been checked that the change in the first six frequencies does not exceed $0.1 \%$. Then a simplified mesh including only 192 CHEXA elements is created. A compromise has been established to keep a satisfying dynamic behaviour and a reasonable number of dofs. Change in the first six frequencies does not exceed $3 \%$ and the simplified mesh is sufficiently fine to avoid shear and volumetric mesh locking due to incompressibility. Consequently reduced integration elements or hybrid elements are not necessary.

Moreover, condensation is applied on the cross part and on the four metallic blocks in order to reduce the number of dofs for simulation. First free mode of cross part and of each metallic block is higher than $1 \mathrm{kHz}$ and exceeds frequency band for simulation $(10-120 \mathrm{~Hz})$. This observation allows to carry out a Guyan condensation [28] for these metallic parts on interfaces with rubber parts. After reduction, the model integrates 1662 dofs and 192 rubber elements. 6 dofs are used to represent the rigid body movement of the satellite instrument.

Finally, an excitation force is applied on the single concentrated mass in order to obtain a significant non linear behaviour of the rubber mounts. A value of $145 \mathrm{~N}$ is chosen in order to assure that the equivalent strain does not exceed $4 \%$ so that mechanical rubber properties remain in the range of experimental data. Indeed, for homogeneous rubber properties corresponding to an experimental dynamic amplitude of 5\% (respectively $0.1 \%$ ), a linear simulation with MSC.Nastran shows that the maximum equivalent strain obtained among all rubber elements (and for all the frequency band) is of $4 \%$ (respectively $2.41 \%$ ). A value of $145 \mathrm{~N}$ will consequently lead to a maximum equivalent strain measure included in this range of $2.41-4 \%$ as desired. 


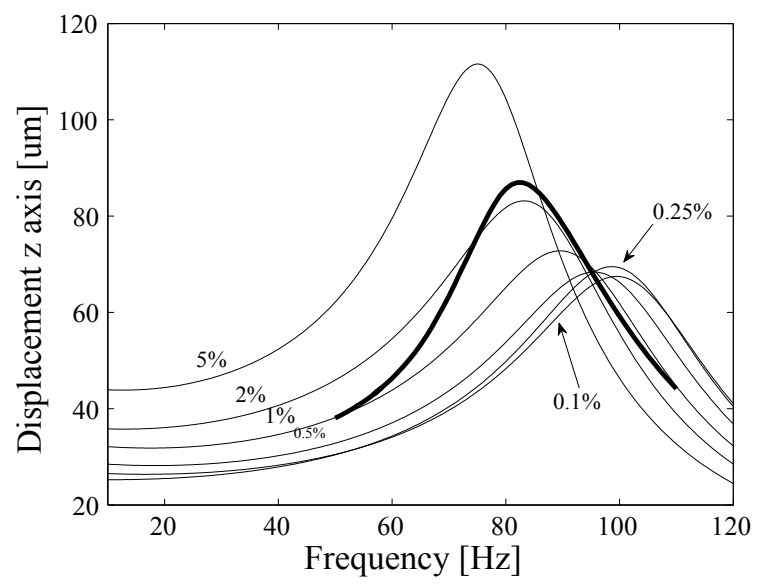

(a)

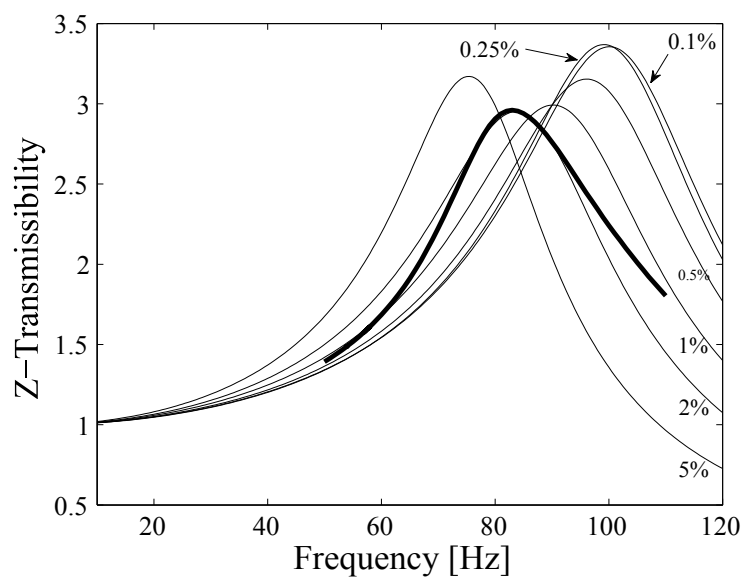

(b)

Fig. 5. (a) Displacement of concentrated mass (b) Force Transmissibility along z-axis $\frac{R z}{F}$ : - homogeneous properties of rubber without amplitude dependency); - non linear procedure with amplitude dependency.

\section{Results}

Simulations are performed on a frequency band of [10-120 Hz] in order to capture the pumping mode. In conjunction with the proposed non linear procedure, linear frequency analyses are carried out with MSC.Nastran with SOL108 algorithm. These calculations consider only frequency dependency on the viscoelastic material and affect homogeneous properties to rubber mounts corresponding to a fixed experimental dynamic amplitude.

\subsection{Displacements}

First results show that resonance of the pumping mode may vary on the frequency band of $[75-100] \mathrm{Hz}$ if homogeneous properties are considered. Indeed, Fig. 5(a) describes the evolution of the displacement along Z-axis of the concentrated mass for the six different dynamic amplitudes for linear simulations. Unsurprisingly, resonance frequency decreases for larger dynamic amplitude. The maximum value of $100 \mathrm{~Hz}$ is obtained for an amplitude of $0.1 \%$ while the minimum of $75 \mathrm{~Hz}$ is obtained for $5 \%$. This decrease is associated with the rubber softening observed on experimental data in Fig. 4. Maximum displacement also increases for larger dynamic amplitudes and varies between 67 and $111 \mu \mathrm{m}$. Concerning the non linear procedure, resonance frequency is $83 \mathrm{~Hz}$ which is an intermediary value showing that rubber is working in its non linear domain. The maximum displacement is $87 \mu \mathrm{m}$ and is coherent with linear calculations. Around resonance, this result is really close to the curve obtained with a homogeneous modulus chosen for an amplitude of $2 \%$. However, it can also be noted that the curve shape is lightly different from linear calculations.

\subsection{Transmissibility}

A short analysis on the force transmissibility on the $\mathrm{z}$-axis shows that the maximum value is around 3 for all simulations. The seven results vary between 2.96 and 3.37. This transmissibility is not constant and reaches a minimum value for a dynamic amplitude of nearly $2 \%$. The non linear procedure is also very close to this value. 


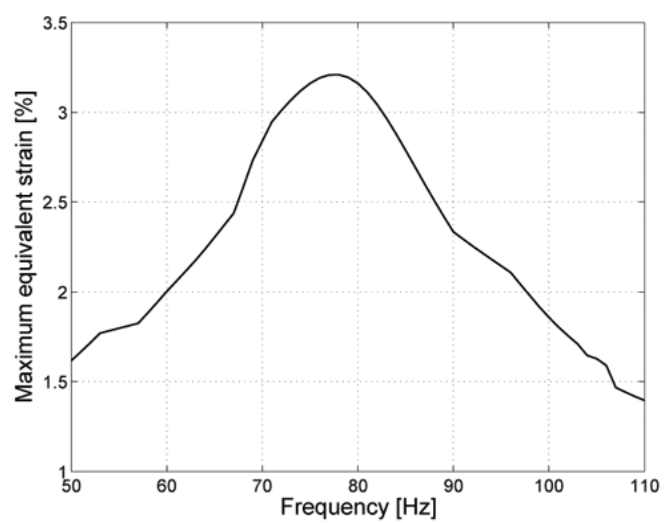

(a)

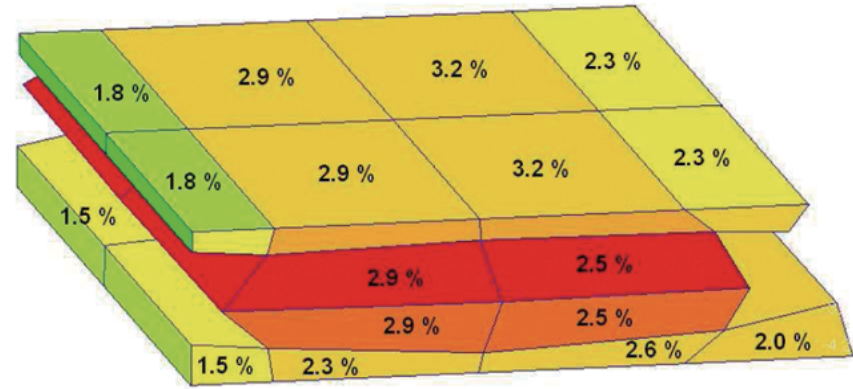

(b)

Fig. 6. Equivalent Deformation: (a) Equivalent strain for element id 50099 (b) Deformed rubber mount and equivalent strain distribution at 83 Hz (figures correspond to equivalent strains and colours to strain energy densities).

\subsection{Equivalent strain}

The proposed procedure is able to deal with non homogeneous properties of rubber and evaluates a different equivalent strain for each element allowing to affect different dynamic properties. This ability is illustrated on Fig. 6(b) where a deformed view of a lower part of a rubber mount is shown. It corresponds to a compression phase. On each element, the equivalent strain is written so the spatial distribution is highlighted. Displayed colours do not correspond to a representation of the equivalent strain but to strain energy density of element surfaces. This view is a typical view from Patran. The maximum strain value of $3.21 \%$ is obtained for element 50099 and for a frequency of $83 \mathrm{~Hz}$, ie for resonance. As expected, this value is included in the range of $2.41-4 \%$. The rubber behaves in its non linear domain. The presented mesh is a simplified mesh and could be improved in order to increase the result precision. However, deformed shape shows that proposed mesh is sufficient to give a satisfactory behaviour of a nearly incompressible material and to avoid volumetric locking. Moreover, the equivalent strain is spatially distributed but also modified for each frequency. Figure 6(a) shows the evolution of the equivalent strain for the element 50099. The maximum value is obtained at resonance.

\section{Conclusion}

The main goal of this study is to propose a new approach in order to take into account Fletcher-Gent effect or Payne effect in a linear viscoelastic rubber material. It allows to perform dynamic simulations of systems integrating rubber parts to predict small vibration amplitude and resonances. No preload are considered here. The proposed methodology provides the ability to consider frequency and amplitude dependency by affecting a different material property to each different rubber material element. It allows to use tabular experimental data from characterization tests which avoids the development of a complex model. Due to the amplitude dependency, a non linear procedure called the harmonic balance method is used to calculate frequency responses. Consequently, an iterative procedure is applied at each frequency to determine vibration amplitudes which are not a priori known. In order to assign an adapted property to each element, an equivalent strain measure is evaluated from an energy balance. This energy balance enables to associate dynamic properties of a material element subjected to multiaxial strain state with experimental dynamic properties of a material sample subjected to an uniaxial strain state. Afterward, shear storage and loss moduli associated with this equivalent strain are deduced from experimental data. Then, the non linear stiffness of rubber elements may be calculated at each iteration. The non linear solver repeats this operation until convergence. Practically, the methodology is developed to deal with models defined with MSC.Nastran. DMAP alter procedures are developed in order to evaluate energies in the model and non linear solver is developed with Matlab. 
The method is then applied on a satellite instrument isolator including four non linear rubber mounts. Results show a non homogeneous distribution of rubber properties since the equivalent strain differs from one element to another. Moreover, it has been shown that the maximum equivalent strain varies between $1.5 \%$ and $3.21 \%$ on a frequency band of [50-110] Hz. The Payne effect also strongly influences resonance frequency. Indeed, the pumping mode is located at nearly $83 \mathrm{~Hz}$ while a procedure considering homogeneous properties may yield a resonance in the band [75-110]Hz.

Finally, the methodology can deal with any configurations of excitation (combination of torsion, shear. ..). In the case of a more pronounced non linearity, more than one harmonic may be chosen for simulations.

\section{Appendix 1: HBM formulation}

In this appendix, the HBM formulation is explained in details with application to the proposed study. We consider the previous governing equation of motion

$$
\mathbf{M} \ddot{X}+\mathbf{D} \dot{X}+\mathbf{K} X=F(X, \dot{X}, \Omega, t)=F_{L}(\Omega, t)+F_{N L}(X, \dot{X}, \Omega, t)
$$

where $\mathbf{M}, \mathbf{K}$ and $\mathbf{D}$ are the mass, stiffness and damping matrices. $\Omega$ is the angular frequency of an external periodic force $F_{L}(\Omega, t)$ applied to the system. $F_{N L}(X, \dot{X}, \Omega, t)$ concerns the nonlinear terms of the mechanical system.

As previously explianed, the non-linear periodic response $X(t)$ of the system can be approximated by finite Fourier series:

$$
\begin{aligned}
& X(t)=B_{0}+\sum_{k=1}^{m}\left(A_{k} \sin \left(\frac{k}{\nu} \Omega t\right)+B_{k} \cos \left(\frac{k}{\nu} \Omega t\right)\right) \\
& X(t)=\left[\mathbf{I} \sin \left(\frac{\Omega}{\nu} t\right) \mathbf{I} \cos \left(\frac{\Omega}{\nu} t\right) \mathbf{I} \ldots \sin \left(\frac{k}{\nu} \Omega t\right) \mathbf{I} \cos \left(\frac{k}{\nu} \Omega t\right) \mathbf{I} \ldots\right]\left[\begin{array}{lllll}
B_{0} & A_{1} B_{1} \ldots & A_{k} & B_{k} & \ldots
\end{array}\right]^{T} \\
& X(t)=\mathbf{T}(t) Z
\end{aligned}
$$

where $\mathbf{I}$ is the $n \times n$ identity matrix, $Z=\left[B_{0} A_{1} B_{1} \ldots A_{k} B_{k} \ldots\right]^{T}$ is the $(2 m+1) n \times 1$ vector containing Fourier coefficients, $m$ is the number of harmonics retained for the truncation, $\nu$ is an integer used to represent possible subharmonics, and $\mathbf{T}(t)=\left[\mathbf{I} \sin \left(\frac{\Omega}{\nu} t\right) \mathbf{I} \cos \left(\frac{\Omega}{\nu} t\right) \mathbf{I} \ldots \sin \left(\frac{k}{\nu} \Omega t\right) \mathbf{I} \cos \left(\frac{k}{\nu} \Omega t\right) \mathbf{I} \ldots\right]$ is the $n \times(2 m+1) n$ matrix containing trigonometric functions.

The same work is then accomplished for the global force $F$ :

$$
F(X, \dot{X}, \Omega, t)=\mathbf{T}(t)\left[C_{0} S_{1} C_{1} \ldots S_{k} C_{k} \ldots\right]^{T}=\mathbf{T}(t) b
$$

In order to compute velocities and accelerations, a frequential derivative operator is defined:

$$
\nabla=\operatorname{diag}\left(\mathbf{0}_{n \times n}, \nabla_{1}, \ldots, \nabla_{m}\right) \text { with } \nabla_{k}=\frac{k}{\nu} \Omega\left[\begin{array}{cc}
\mathbf{0} & -\mathbf{I} \\
\mathbf{I} & \mathbf{0}
\end{array}\right]
$$

Thus one may write:

$$
\dot{X}(t)=\mathbf{T}(t) \nabla Z \text { and } \ddot{X}(t)=\mathbf{T}(t) \nabla^{2} Z
$$

By replacing Eqs (21) and (24) into Eq. (20), one obtains:

$$
\mathbf{M T}(t) \nabla^{2} Z+\mathbf{D T}(t) \nabla Z+\mathbf{K T}(t) Z=\mathbf{T}(t) b
$$

Considering that for a $n \times n$ matrix $\mathbf{W}$ and a $(2 m+1) n \times 1$ vector $Y$ :

$$
\mathbf{W T}(t) Y=\mathbf{T}(t) \mathbf{N}_{\mathbf{W}} Y=\mathbf{T}(t) \operatorname{diag}(\mathbf{W}, \mathbf{W}, \ldots) \underset{\times(2 m+1) n}{\underset{\times(2 m+1) n}{(2 m+1) n}} Y
$$

Equation (25) becomes:

$$
\mathbf{T}(t)\left(\mathbf{N}_{\mathbf{M}} \nabla^{2}+\mathbf{N}_{\mathbf{D}} \nabla+\mathbf{N}_{\mathbf{K}}\right) Z=\mathbf{T}(t) b
$$


Time dependency may be suppressed and a frequency algebraic equation linking Fourier coefficient may be obtained using a Galerkin method which is a projection of the equation on trigonometric functions. Indeed these trigonometric functions define a scalar product:

$$
<f, g>=\frac{1}{T} \int_{0}^{T} f(t) g(t) d t
$$

Thus one may write:

$$
\frac{1}{T} \int_{0}^{T}{ }^{T} \mathbf{T}(t) \mathbf{T}(t) d t=\frac{1}{2}\left[\begin{array}{ccc}
2 \mathbf{I} & & \mathbf{0} \\
& \mathbf{I} & \\
& \mathbf{I} & \\
& & \ddots
\end{array}\right]=\mathbf{L}_{\begin{array}{l}
(2 m+1) n \\
\times(2 m+1) n
\end{array}}
$$

Applying this scalar product on Eq. (27) leads to a $(2 m+1) * n$ equation system:

$$
\mathbf{A} Z=b \text { with } \mathbf{A}=\mathbf{N}_{\mathbf{M}} \nabla^{2}+\mathbf{N}_{\mathbf{D}} \nabla+\mathbf{N}_{\mathbf{K}}
$$

A may be expressed in a simpler manner:

$$
\mathbf{A}=\left[\begin{array}{ccccccc}
\mathbf{K} & & & & & & \\
& \mathbf{K}-\left(\frac{\Omega}{\nu}\right)^{2} \mathbf{M} & -\frac{\Omega}{\nu} \mathbf{D} & & & & \\
& \frac{\Omega}{\nu} \mathbf{D} & \mathbf{K}-\left(\frac{\Omega}{\nu}\right)^{2} \mathbf{M} & & & & \\
& & & \ddots & & & \\
& & & & \mathbf{K}-\left(\frac{k}{\nu} \Omega\right)^{2} \mathbf{M} & -\frac{k}{\nu} \Omega \mathbf{D} & \\
& & & & \frac{k}{\nu} \Omega \mathbf{D} & \mathbf{K}-\left(\frac{k}{\nu} \Omega\right)^{2} \mathbf{M} & \\
& & & & & & \ddots
\end{array}\right]
$$

This system is equivalent of finding zeros of a function $H: \mathbb{R}^{(2 m+1) \times n} \rightarrow \mathbb{R}^{(2 m+1) \times n}$ :

$$
H(Z)=\mathbf{A}(\Omega) Z-b(Z, \Omega)=\mathbf{A}(\Omega) Z-b_{L}(\Omega)-b_{N L}\left(Z_{v}, \Omega\right)
$$

We note that $b$ is dependent on $Z$ and $\Omega$ because $b$ corresponds to the Fourier coefficients of $F(X, \dot{X}, \Omega, t)$. $b$ is a sum of Fourier coefficients from the linear excitation force $b_{L}$ and from non linear force $b_{N L} . b_{N L}$ depends only on the Fourier coefficients $Z_{v}$ of the $v$ dofs linked with the viscoelastic elements.

In the case where no analytical expression may be written between $b_{N L}$ and $Z_{v}$, an evaluation of the approximate temporal terms $X(t)$ and $F_{N L}(t)$ combining FFT procedures allows to numerically evaluate the Fourier coefficients $b_{N L}$ for each iteration.

Concerning Payne effect in viscoelastic materials, it has been described that, within certain strain amplitude limit, higher-order terms are not significant in the material response [21]. Consequently, a single harmonic has been retained in the HBM procedure and $\nu=1$ leading to a $2 n \times 2 n$ equation system:

$$
\left[\begin{array}{cc}
\mathbf{K}-\Omega^{2} \mathbf{M} & -\Omega \mathbf{D} \\
\Omega \mathbf{D} & \mathbf{K}-\Omega^{2} \mathbf{M}
\end{array}\right]\left[\begin{array}{l}
A_{1} \\
B_{1}
\end{array}\right]=b_{L}(\Omega)+b_{N L}\left(Z_{v}, \Omega\right)
$$

\section{References}

[1] D.L. Kunz, Elastomer modelling for use in predicting helicopter lag damper behavior, Journal of Sound and Vibration 226(3) (1999), 585-594.

[2] T. Bruger and M. Rabkin, FEM-Simulation of comfort relevant properties of elastorneric mountings: The Payne-effect under preloading, In Constitutive Models for Rubber V, 2008, pages 439-442. 5th European Conference on Constitute Models for Rubber, Paris, FRANCE, SEP 04-07, 2007.

[3] A.I. Medalia, Effect of Carbon-Black on Dynamic Properties of Rubber Vulcanizates, Rubber Chemistry and Technology 51(3) (1978), 437-523. 
[4] M.J. Wang, Effect of polymer-filler and filler-filler interactions on dynamic properties of filled vulcanizates, Rubber Chemistry and Technology 71(3) (1998), 520-589.

[5] L. Mullins, Softening of rubber by deformation, Rubber Chenmistry and Technology 42 (1969), 339-362.

[6] A.R. Payne, Low strain dynamic properties of filled rubbers, Rubber Chemistry and Technology 44 (1971), $440-478$.

[7] W.P. Fletcher and A.N. Gent, Non-linearity in the dynamic properties of vulcanisaed rubber compounds, I.R.I. Transactions 29 (1953), $266-280$.

[8] L. Chazeau, J.D. Brown, L.C. Yanyo and S.S. Sternstein, Modulus recovery kinetics end other insights into the Payne effect for filled elastomers, POLYMER COMPOSITES 21(2) (2000), 202-222.

[9] J.D. Ferry, ViscoelasticProperties of Polymers, Wiley, New-York, third ed. edition, 1980.

[10] N. Gil-Negrete, On the Modelling and Dynamic Stiffness Prediction of Rubber Isolators, PhD thesis, University of Navarra, Spain, 2004.

[11] J. Lubliner, A Model of Rubber Viscoelasticity, Mechanics Research Communications 12(2) (1985), 93-99.

[12] M. Enelund and P. Olsson, Damping described by fading memory - analysis and application to fractional derivative models, International Journal of Solids and Structures 36(7) (1999), 939-970.

[13] G. Kraus, Mechanical losses in carbon-black filled rubbers, Journal of Applied Polymer Science, Applied Polymer Symposium 39 (1984).

[14] J.D. Ulmer, Strain dependence of dynamic mechanical properties of carbon black-filled rubber compounds, Rubber Chemistry and Technology 69(1) (1996), 15-47.

[15] M. Berg, A non-linear rubber spring model for rail vehicle dynamics analysis, Vehicle System Dynamics 30(3-4) (1998), 197-212.

[16] M. Kaliske and H. Rothert, Constitutive approach to rate-independent properties of filled elastomers, International Journal of Solids and Structures 35(17) (1998), 2057-2071.

[17] M. Rabkin, T. Bruger and P. Hinsch, Material model and experimental testing of rubber components under cyclic deformation, In Proceedings of the Third Conference on Constitutive Models for Rubbers, 2003, pages 319-324.

[18] A. Austrell, A.K. Olsson and M. Jonsson, A method to analyse the non-linear dynamic behaviour of carbon-black filled rubber components using standard fe codes. In Proceedings of the Second Conference on Constitutive Models for Rubbers, 2001, pages 231-235.

[19] N. Gil-Negrete, J. Vinolas and L. Kari, A simplified methodology to predict the dynamic stiffness of carbon-black filled rubber isolators using a finite element code, Journal of Sound and Vibration 296(4-5) (2006), 757-776.

[20] M. Rabkin, Simulation of the Fletcher-Gent effect by using of the subroutine UPHI MSC/MARC (c), In Constitutive Models for Rubber $V$, 2008, pages 263-268. 5th European Conference on Constitute Models for Rubber, Paris, FRANCE, SEP 04-07, 2007.

[21] C.M. Roland, Dynamic Mechanical-Behavior of Filled Rubber at Small Strains, Journal of Rheology 34(1) (1990), 25-34.

[22] E.H. Boutyour, E.M. Daya, L. Azrar and M. Potier-Ferry, An approximated harmonic balance method for nonlinear vibration of viscoelastic structures, Journal of Engineering Materials and Technology 128(3) (2006), 330-335.

[23] M. Bilasse, E.M. Daya and L. Azrar, Linear and nonlinear vibrations analysis of viscoelastic sandwich beams, Journal of Sound and Vibration 329(23) (2010), 4950-4969.

[24] V. Jaumouillé, J.-J. Sinou and B. Petitjean, An adaptive harmonic balance method for predicting the nonlinear dynamic responses of mechanical systems-Application to bolted structures, Journal of Sound and Vibration 329(19) (September 2010), $4048-4067$.

[25] N. Coudeyras, J.-J. Sinou and S. Nacivet, A new treatment for predicting the self-excited vibrations of nonlinear systems with frictional interfaces: The constrained harmonic balance method, with application to disc brake squeal, Journal of Sound and Vibration 319(3-5) (2009), 1175-1199.

[26] N. Coudeyras, S. Nacivet and J.-J. Sinou, Periodic and quasi-periodic solutions for multi-instabilities involved in brake squeal, Journal of Sound and Vibration 328(4-5) (2009), 520-540.

[27] J.-J. Sinou, Effects of a crack on the stability of a non-linear rotor system, International Journal of Non-Linear Mechanics 42(7) (2007), 959-972.

[28] R.J. Guyan, Reduction of Stiffness and Mass Matrices, AIAA Journal 3(2) (1965). 

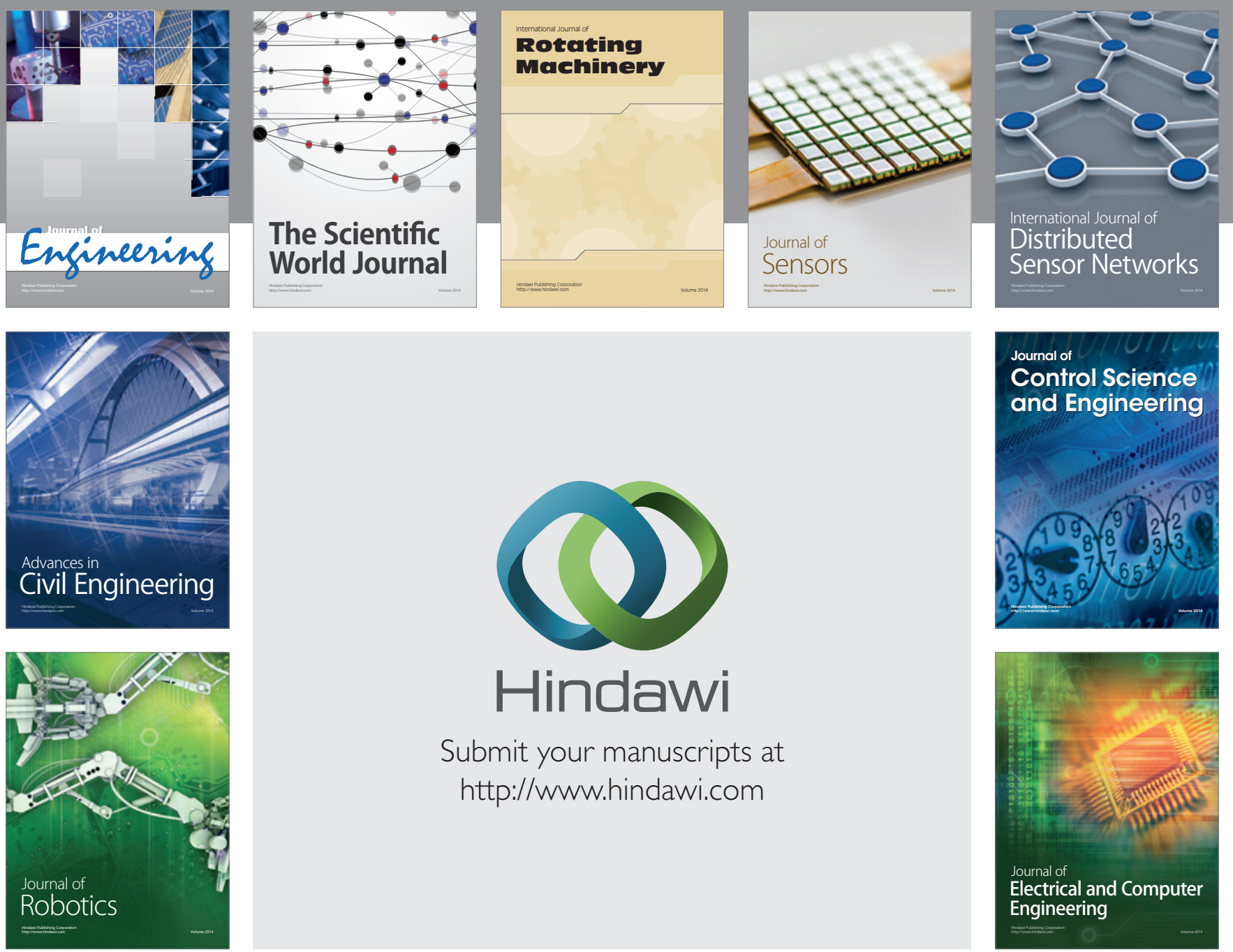

Submit your manuscripts at

http://www.hindawi.com
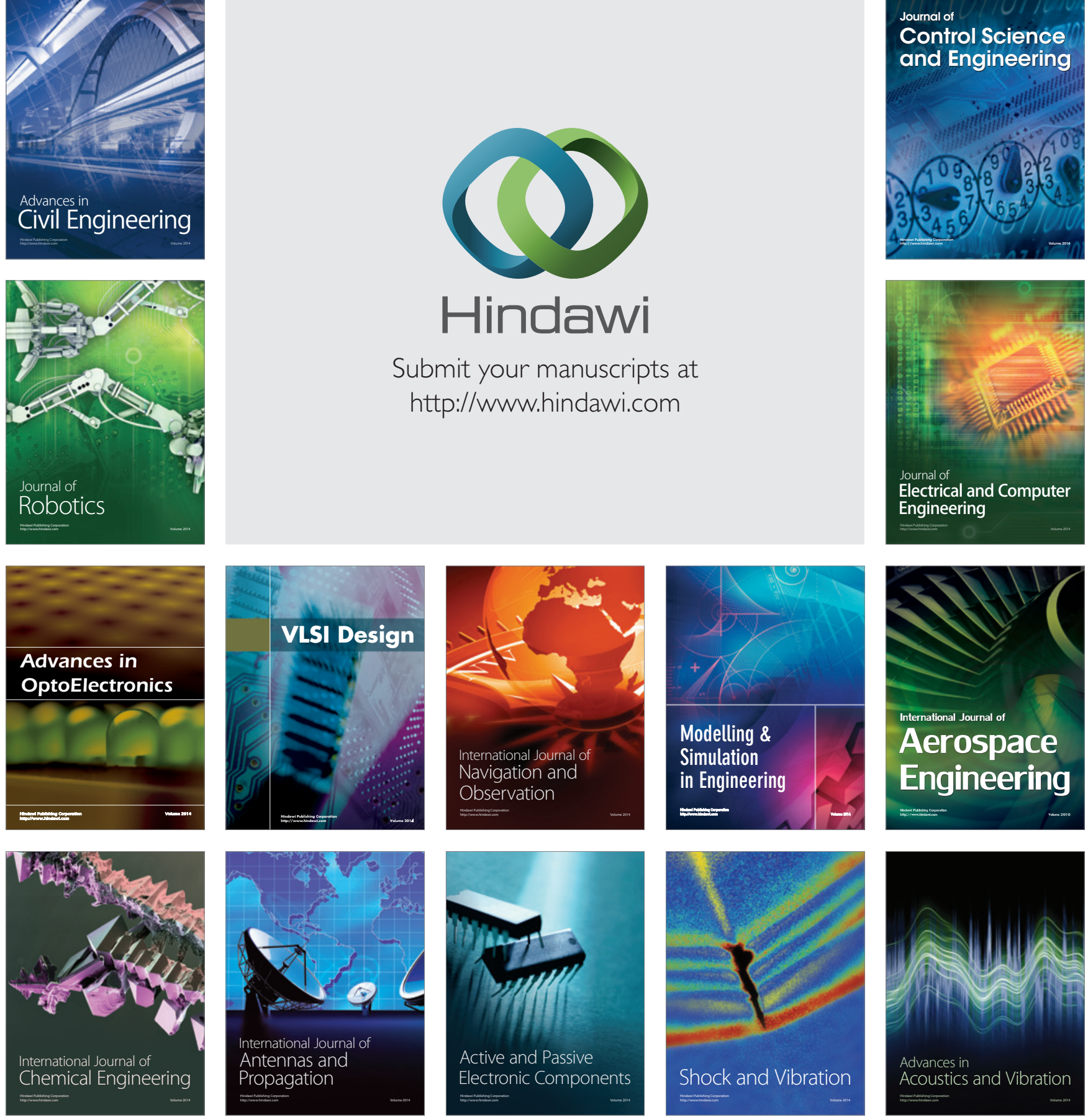